\title{
Input and Output Gain Modulation by the Lateral Interhemispheric Network in Early Visual Cortex
}

\author{
Thomas Wunderle, ${ }^{1,2}$ David Eriksson, ${ }^{1,2}$ Christiane Peiker, ${ }^{1}$ and ${ }^{\circ}$ Kerstin E. Schmidt ${ }^{1,3}$ \\ ${ }^{1}$ Max Planck Institute for Brain Research, 60528 Frankfurt, Germany, ${ }^{2}$ Ernst Strüngmann Institute (ESI) for Neuroscience in Cooperation with Max Planck \\ Society, 60528 Frankfurt, Germany, and ${ }^{3}$ Brain Institute, Federal University of Rio Grande do Norte, 59056-450 Natal, Brazil
}

\begin{abstract}
Neurons in the cerebral cortex are constantly integrating different types of inputs. Dependent on their origin, these inputs can be modulatory in many ways and, for example, change the neuron's responsiveness, sensitivity, or selectivity. To investigate the modulatory role of lateral input from the same level of cortical hierarchy, we recorded in the primary visual cortex of cats while controlling synaptic input from the corresponding contralateral hemisphere by reversible deactivation. Most neurons showed a pronounced decrease in their response to a visual stimulus of different contrasts and orientations. This indicates that the lateral network acts via an unspecific gain-setting mechanism, scaling the output of a neuron. However, the interhemispheric input also changed the contrast sensitivity of many neurons, thereby acting on the input. Such a contrast gain mechanism has important implications because it extends the role of the lateral network from pure response amplification to the modulation of a specific feature. Interestingly, for many neurons, we found a mixture of input and output gain modulation. Based on these findings and the known physiology of callosal connections in the visual system, we developed a simple model of lateral interhemispheric interactions. We conclude that the lateral network can act directly on its target, leading to a sensitivity change of a specific feature, while at the same time it also can act indirectly, leading to an unspecific gain setting. The relative contribution of these direct and indirect network effects determines the outcome for a particular neuron.
\end{abstract}

Key words: contrast gain; corpus callosum; lateral connection; primary visual cortex; response gain; reversible deactivation

\section{Introduction}

Information processing in the CNS relies on a combination of various signal streams. Already in primary sensory cortex, the local microcircuit is fed by long-range intracortical, feedback, and callosal input (Gilbert and Wiesel, 1979; Innocenti, 1994; Angelucci and Bressloff, 2006). What remains unclear is how these inputs are combined in the cortex. Although a lot is known about the integrative properties of single neurons in vitro, much less is known about the neuronal arithmetic of neurons embedded in their network in vivo (Silver, 2010). Previously, we demonstrated that the combination of ipsilateral and contralateral inputs in primary visual cortex leads to a context-dependent scaling of orientation tuning curves (Wunderle et al., 2013). This could be due to a specific interaction of the callosal input with one or more of the mechanisms creating orientation selectivity or a more general phenomenon of cortical processing. However, the nature of the modulation by intracortical inputs can be inferred by observing a neuron's contrast response function (CRF).

Received Oct. 7, 2014; revised Feb. 20, 2015; accepted March 23, 2015.

Author contributions: T.W. and K.E.S. designed research; T.W., D.E., C.P., and K.E.S. performed research; T.W. analyzed data; T.W. and K.E.S. wrote the paper.

This work was supported by the Max Planck Society. We are grateful to Sergio Neuenschwander for the acquisition system and to Danko Nikolic for the stimulus interface.

The authors declare no competing financial interests.

Correspondence should be addressed to Thomas Wunderle, Ernst Strüngmann Institute for Neuroscience (ESI) in Cooperation with Max Planck Society, Deutschordenstraße 46, 60528 Frankfurt, Germany. E-mail: thomas.wunderle@esi-frankfurt.de.

DOI:10.1523/JNEUROSCI.4154-14.2015

Copyright $\odot 2015$ the authors $\quad 0270-6474 / 15 / 357682-13 \$ 15.00 / 0$
Changes in the neuron's responsivity shift the response vertically along the response axis ("response gain"), which corresponds to a linear output increase, i.e., a multiplicative scaling of the neuron's evoked response. On the other hand, changes in the neuron's sensitivity shift the response horizontally along the contrast axis ("contrast gain"), which corresponds to a multiplicative scaling of the input, i.e., the stimulus contrast (Fig. 1). On the level of microcircuits, it has been shown that neuromodulators (Bhattacharyya et al., 2013) and inhibitory neurons can control the gain of pyramidal neurons at the input or output (Katzner et al., 2011; Wilson et al., 2012). Similarly, for more complex functions like surround suppression (Cavanaugh et al., 2002) and, moreover, shifts in spatial attention (Reynolds et al., 2000; Williford and Maunsell, 2006), such changes in input and output gain have been described previously. Here, we investigate these mechanisms for the actions of interhemispheric connections.

In early visual cortex, callosal connections link the two hemispheres at the border between the areas 17 and 18 (Innocenti, 1994) and are discussed to extend the lateral network at the same level of cortical hierarchy across the vertical meridian (Schmidt, 2013) as long-range intrinsic connections do across the horizontal meridian (Jeffs et al., 2009). To unveil the scaling mechanism of these lateral interhemispheric inputs, we combined extracellular recordings in the primary visual cortex of anesthetized cats with reversible thermal deactivation. We recorded in the callosal recipient zone while deactivating locally the corresponding callosal sending zone in the other hemisphere (Fig. 1). 

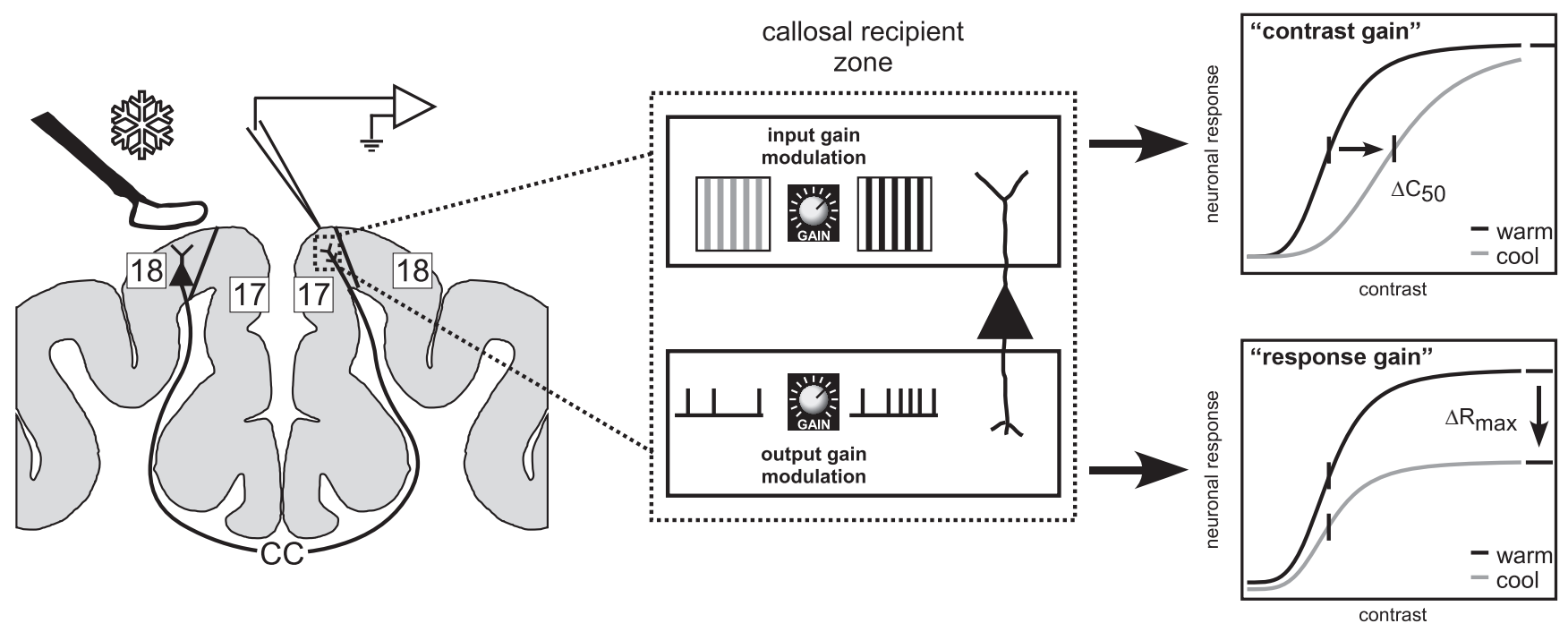

Figure 1. Left, Experimental setup. Neurons projecting to the contralateral hemisphere were reversibly deactivated by local cooling while spiking activity of neurons in the callosal recipient zone was recorded extracellularly. Middle, Callosal connections (CC) could modulate the gain at a neuron's input or its output. Right, Input gain modulation is expressed as a change in the semisaturation contrast $\left(C_{50}\right)$ of a neuron's contrast response function, shifting the curve horizontally. Output gain modulation is expressed as a change in the maximal response $\left(R_{\max }\right)$ of a neuron, shifting the contrast response curve vertically.

We found that lateral input conveyed via the corpus callosum can change the contrast sensitivity and responsivity of a neuron, i.e., modulate the gain at the input or output, or a combination of both. We propose that the relative contribution of direct interhemispheric and indirect intrinsic inputs determines the scaling mechanism for each particular neuron. A simple model that includes contrast-dependent response normalization can account for the experimental results.

\section{Materials and Methods}

All procedures in this study were approved by the ethics committee of the State of Hessen in accordance with the guidelines of the German law for the protection of animals.

Surgical procedures and recordings. A detailed description of the surgical and recording procedures can be found in the studies by Peiker et al. (2013) and Wunderle et al. (2013). For this study, five adult cats (one male, four females) bred in the Institute's colony were used. After initial anesthesia (10 mg/kg ketamine supplemented with $1 \mathrm{mg} / \mathrm{kg}$ xylazine), two craniotomies were performed around the area 17/18 border region (Horsley-Clarke coordinates AP +4 to -5 , ML +1 to +7 ) on both hemispheres in topographical correspondence. A cryoloop with a dimension of $5 \times 3 \mathrm{~mm}^{2}$ giving rise to a deactivated region of $\sim 8 \times 6 \mathrm{~mm}^{2}$ was placed on the left hemisphere. On the right hemisphere, two $4 \times 4$ tungsten microelectrode arrays (planar configuration, fixed electrode length, $250 \mu \mathrm{m}$ spacing, $1 \mathrm{M} \Omega$, Microprobes) were positioned in topographical correspondence to the cryoloop to record multiunit activity. We inserted the electrode arrays and started to collect data as soon as most of the channels demonstrated visually driven activity. After some recording sessions, we advanced the arrays to isolate new multiunits. According to this procedure and the characteristics of the encountered receptive fields (RFs), it is likely that the majority of our units stem from supragranular layers. However, due to the curvature of the brain, the laminar position of neurons recorded with our arrays could not be determined with precision. Therefore, in one animal, two additional 16 contact laminar silicone probes (Neuronexus) were positioned in area 17 close to the $17 / 18$ border. We used optical imaging of intrinsic signals to confirm the location of the 17/18 border before electrode implantation (Wunderle et al., 2013). Based on RF size and position as well as spatial and temporal frequency preference, we classified the recorded multiunits ( $n=142$ ) as being located in the $17 / 18$ transition zone or more toward area 17 or area 18 (Movshon et al., 1978; Tusa et al., 1979). According to this classification, the majority of recorded sites $(n=88)$ were located at the $17 / 18$ border close to the vertical meridian, in area $17(n=49)$, and a few in area $18(n=5)$.

For recordings, the animals were paralyzed (pancuronium bromide, $0.15 \mathrm{mg} / \mathrm{kg} / \mathrm{h}$ ), and anesthesia was maintained with $0.6 \%$ halothane and $\mathrm{N}_{2} \mathrm{O} / \mathrm{O}_{2}(70 / 30 \%)$. A recording session consisted of three states: warm (precooling), cooling, and rewarm (recovery). Recording during the cooling state was initiated after the cooling loop reached a stable temperature of $3 \pm 1.5^{\circ} \mathrm{C}$ for $5 \mathrm{~min}$. The recovery recording was started $\sim 20 \mathrm{~min}$ after cooling was terminated.

Visual stimulation. We presented drifting square wave gratings on a gamma-corrected 21 inch CRT monitor at a $57 \mathrm{~cm}$ distance from the animal's eyes, which were fitted with contact lenses to ensure optimal refraction. Whole-field gratings were presented at seven different contrasts $(4,8,12,18,24,48$, and $100 \%$ Michelson contrast) drifting in eight directions $\left(45^{\circ}\right.$ steps) orthogonal to their orientation. We started data collection $250 \mathrm{~ms}$ before stimulus onset (blank). Stimuli moved for 750 $\mathrm{ms}$ immediately after onset. We later added the average (spontaneous) activity in the $250 \mathrm{~ms}$ preceding each condition to the CRFs representing the response at $0 \%$ contrast. Spatial frequency and speed of the gratings were fixed for a given recording session and chosen from two sets, depending on which set was preferred by most of the neurons. The parameters for the area 17 set were 0.5 cycles per degree and $4 \%$ s, and for the area 18 set 0.15 cycles per degree and $16 \%$ s. Each condition was randomly presented 15 times with an interstimulus interval of $1.5 \mathrm{~s}$.

Data analysis. Offline analysis was performed using custom-written software in Matlab (MathWorks). From 32-64 simultaneously recorded channels, only those exhibiting reliable multiunit activity were used for further analysis according to the following criteria: (1) A three-factor ANOVA was performed to test whether the response amplitude (spikes per second) to a drifting stimulus was significantly larger than within the prestimulus period (blank), if there was a significant difference between different stimulus directions and also between different contrasts (all three $p<0.05$ ). (2) The direction or orientation tuning of the multiunits reached a certain threshold. We calculated a direction selectivity index (DSI) and orientation selectivity index (OSI), defined as 1 minus the circular variance of the direction or orientation tuning curve, respectively (Swindale, 1998). Those indices range from 0 (totally unselective) to 1 (perfectly selective). As some analyses tested effects on direction and orientation tuning curves (see Fig. 4), only neurons with a DSI or OSI of $>0.2$ were considered for further analysis. We classified neurons as simple or complex based on their response modulation to the drifting gratings (Skottun et al., 1991). Neurons were defined as complex if their 
response at the fundamental frequency (F1) of the drifting grating was smaller than the mean firing rate (F0). Only two multiunits were classified as "simple" by this method; all others had an F1/F0 ratio smaller than one. We also applied a spike-sorting algorithm (wave_clus 2.0, Quiroga et al., 2004) to obtain single units. In addition to visual inspection, a cluster was defined as a single unit if its signal-to-noise ratio was larger than 6 (peak to trough distance divided by the average SD) and if no more than $10 \%$ of the spikes had an interspike interval smaller than $10 \mathrm{~ms}$. Applying these criteria revealed 30 single units with a reasonable fit to the CRFs out of 142 recorded multiunits.

RFs were mapped throughout the experiment using an automatic back-projection method (Fiorani et al., 2013). Briefly, the spiking activity in response to a bar (width, $1^{\circ}$; length, complete mapping area of $30 \times$ $30^{\circ}$ ), drifting in 16 different directions (speed, $20^{\circ} / \mathrm{s}$ ), was mapped to visual space and summed across directions. From the resulting 2D density plot, we defined the area above $70 \%$ of the maximum response as the border of the RF. The center of the RF was determined as the centroid of this region. With this method, the absolute size of the RF is just an estimate and depends on the chosen threshold. However, comparisons between sessions indicated that reliability and the sizes of the RFs were in good agreement with reports in the literature for the areas we recorded in (Hubel and Wiesel, 1967).

Error bars in the figures denote \pm 1 SEM unless stated otherwise, and significance levels are indicated by asterisks.

Description of responses to contrasts. To describe the behavior of neurons to different contrasts and experimental manipulations, we fitted the neuronal responses with hyperbolic ratio functions (Albrecht and Hamilton, 1982):

$$
R=R_{\max }\left(\frac{c^{n}}{c^{n}+c_{50}^{n}}\right)+s,
$$

where $R$ is the response of the neurons to a stimulus contrast $c$. Four parameters describe the shape of the function: $R_{\max }$ is the maximal (saturation) firing rate, $C_{50}$ is the semisaturation contrast where responses are half-maximal, $n$ determines the slope of the hyperbolic function, and $s$ is level of spontaneous activity. We used a nonlinear optimization algorithm ("trust-region-reflective" implemented in the Matlab function "Isqnonlin") to minimize the sum of squares between the data and the model. The quality of the fit was assessed by calculating an adjusted $R^{2}$ controlling for the number of model parameters.

To get a direct impression of the change in $R_{\max }$ and $C_{50}$ per recorded unit, we performed a combined fit to the data during warm and cooling, keeping $n$ and $s$ fixed between the two experimental states, but allowing $R_{\max }$ and $C_{50}$ to vary independently.

$$
\begin{aligned}
& R_{\text {warm }}=R_{\max , 1}\left(\frac{c^{n}}{c^{n}+c_{50,1}^{n}}\right)+s, \\
& R_{\text {cool }}=R_{\max , 2}\left(\frac{c^{n}}{c^{n}+c_{50,2}^{n}}\right)+s .
\end{aligned}
$$

To compare the changes in $R_{\max }$ and $C_{50}$ between experimental states, we computed a modulation index (MI):

$$
\mathrm{MI}=\left(\frac{P_{\text {cool }}-P_{\text {warm }}}{P_{\text {cool }}+P_{\text {warm }}}\right),
$$

where $P_{\text {cool }}$ and $P_{\text {warm }}$ are the parameters $R_{\max }$ or $C_{50}$ during the cooling and warm states, respectively. A negative value of the MI indicates a decrease of the parameter, whereas a positive value indicates an increase. Confidence intervals (CIs) for each parameter were determined by bootstrap resampling (95\% CIs, 1000 resamplings).

The analysis above quantifies a change in the parameters $R_{\max }$ and $C_{50}$, but does not quantify the respective gain mechanism directly. Therefore, we fitted three extended versions of model (1) to the data. The full model included two additional parameters $a_{1}$ and $a_{2}$ to allow for a change in response during thermal deactivation:

$$
R=a_{1} \times\left[R_{\max }\left(\frac{c^{n}}{c^{n}+a_{2} \times c_{50}^{n}}\right)+s\right] .
$$

$a_{1}$ scales the maximal response, thereby shifting the curve vertically ("response gain"). Because of its common usage, we call this type of modulation, including spontaneous activity, response gain, however, it was referred to as "activity gain" in other publications (Williford and Maunsell, 2006). The second parameter, $a_{2}$, changes the semisaturation contrast $\left(C_{50}\right)$, thereby shifting the curve horizontally on a logarithmic contrast axes ("contrast gain"). We performed a combined fit, holding the parameters $R_{\max }, C_{50}, n$, and $s$ fixed between the two experimental states (warm and cool). The response during cooling was fit with the two additional parameters $a_{1}$ and $a_{2}$ being free, and held constant at 1 for the warm state. Additionally, the data were also fitted with two reduced models including only the parameter $a_{1}$ (response gain model) or $a_{2}$ (contrast gain model).

To determine the performance of the two reduced models in comparison to the full one, we calculated a contrast ratio between the residual sum of squares (RSS) of the models:

$$
\mathrm{RMP}=\left(\frac{\mathrm{RSS}_{\text {full }}-\mathrm{RSS}_{\text {red }}}{\mathrm{RSS}_{\text {full }}+\mathrm{RSS}_{\text {red }}}+1\right) \times 100 .
$$

Reduced model performance (RMP) ranges from 0 to 100 , where 0 indicates a low performance of the reduced model, and 100 indicates that the reduced model is as good as the full model in describing the data.

However, to be able to correlate the gain mechanism with other neuronal properties, we calculated a gain index (GI), indicating whether a given unit follows more a contrast or a response gain mechanism. To this end, we directly compared the residual sum of squares between the reduced models:

$$
\mathrm{GI}=\left(\frac{\mathrm{RSS}_{\mathrm{CG}}-\mathrm{RSS}_{\mathrm{RG}}}{\mathrm{RSS}_{\mathrm{CG}}+\mathrm{RSS}_{\mathrm{RG}}}\right),
$$

where $\mathrm{RSS}_{\mathrm{CG}}$ and $\mathrm{RSS}_{\mathrm{RG}}$ are the residual sums of squares for the contrast and response gain models, respectively. The GI ranges from -1 , indicating a contrast gain, to +1 , indicating a response gain.

Description of responses to orientation. We also analyzed orientation tuning at each contrast. To get a better resolution in the orientation domain, we first interpolated average responses to 16 directions using piecewise cubic interpolation as in our previous manuscript (Wunderle et al., 2013). Subsequently, opposite directions were averaged to get orientation tuning curves. Before averaging tuning curves, we aligned responses to each unit's preferred orientation.

We fitted individual tuning curves with Gaussian functions in order to describe their shape, analogous to the fitting of the contrast response functions:

$$
R(\theta)=B+A e^{-\theta-\theta_{p}^{2} /\left(2 \sigma^{2}\right)} .
$$

In the above expression, $R$ is the neurons response to stimulus orientation $\theta$. The function has four free parameters: $\theta_{\mathrm{p}}$ is the neurons preferred orientation given in angular values between $-90^{\circ}$ and $+90^{\circ}$ by \langle\rangle$=$ $\bmod \left(90+\theta-\theta_{\mathrm{p}}, 180\right)-90 ; B$ is the baseline firing rate; $A$ is the amplitude of the tuning curve above baseline; and the parameter $\sigma$ is the tuning width expressed as the SD of the Gaussian function. To compare the parameters between warm and cool, we computed a modulation index according to Equation 4.

\section{Results}

We present here data from 142 multiunits recorded close to the area $17 / 18$ border of the visual cortex from five anesthetized cats. We calculated each unit's CRF from the average multiunit activity during the presentation of an optimal grating.

The average normalized CRFs in the warm, cool, and rewarm states are depicted in Figure $2 A$. The average spike rate decreased significantly during cooling ( $t$ test, $p<0.001$, uncorrected) and recovered during rewarming for all contrasts tested. Next, each 
A

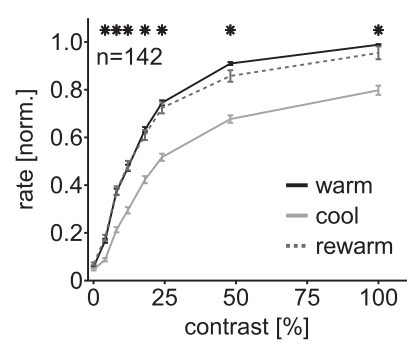

C

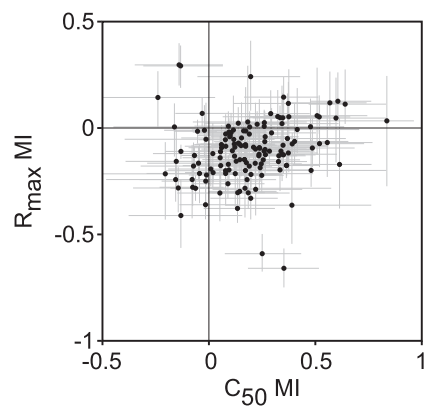

B
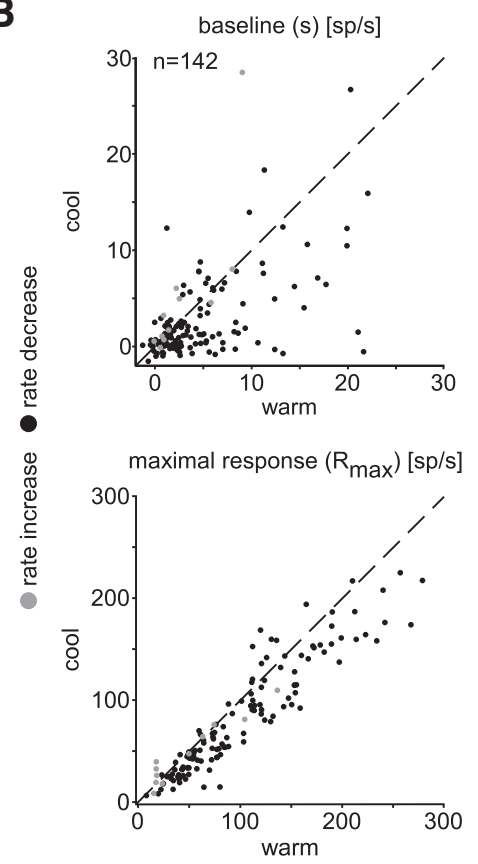

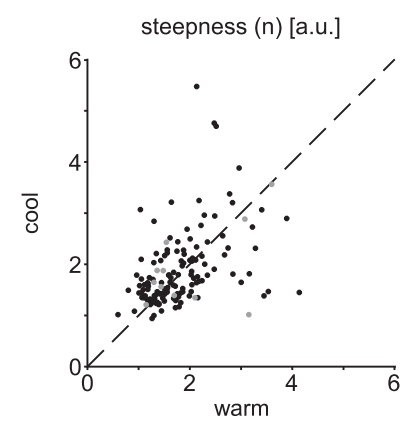

semi-saturation contrast $\left(\mathrm{C}_{50}\right)$ [\%contr]

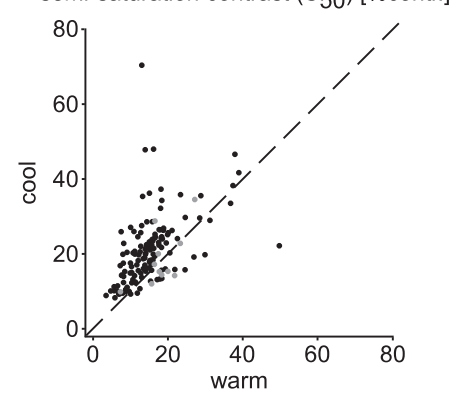

Figure 2. Shape of contrast response functions before and during thermal deactivation. $\boldsymbol{A}$, Average normalized spike rate as a function of grating contrast for the optimal orientation of each multiunit. The activity decreases during cooling for all contrasts and recovers to precooling rates after rewarming. $\boldsymbol{B}$, Impact of thermal deactivation on the four parameters describing the contrast response function for each recording site. Black dots indicate multiunits decreasing their average firing rate; gray dots indicate those increasing their firing rate during callosal blockage. $\boldsymbol{C}$, Modulation indices for $R_{\max }$ and $C_{50}$. Negative values indicate a decrease of the parameter during cooling. Gray bars denote $95 \%$ confidence intervals. ${ }^{*} p<0.05$.

CRF was fit with a hyperbolic ratio function to obtain the curve's characteristic parameters, described in Materials and Methods. This was done before (warm) and during (cool) local thermal deactivation on the hemisphere contralateral to the recordings. The parameter values for the warm and cooled states are plotted against each other in Figure $2 B$.

We separately plotted the data for those recording sites with an average rate increase (Fig. $2 B$, gray dots) and decrease (black dots) across all contrasts. In agreement with our previous studies (Schmidt et al., 2010; Wunderle et al., 2013), only a few neurons increased their activity upon thermal deactivation $(n=11,7.7 \%$ of total multiunits).

The baseline parameter $(s)$, corresponding to the spontaneous activity of the recorded neurons, decreased on average by $32 \%$ (median, Wilcoxon signed-rank test, $p<0.0001$ ). This strong effect, however, resulted mainly from the multiunits with a high spontaneous rate (median split; mean rate, 7.9 spikes/s), whereas for multiunits with a low spontaneous rate (mean rate, 1.2 spikes/s) the decrease was only $9 \%$, consistent with what we reported previously (Wunderle et al., 2013). The steepness parameter ( $n$; slope of the fitted CRF) was, on average, not affected by interhemispheric input (median change, $+3.5 \%$; $p=0.22$ ). The maximal response of the multiunits $\left(R_{\max }\right)$ decreased significantly by $19 \%$ (median; $p<0.0001$ ). Interestingly, for some recording sites ( $n=24$ or $17 \%$ ), $R_{\max }$ increased during thermal deactivation, although the average firing rate across contrasts decreased (for 18 of the 24). This is because those CRFs became nonsaturating, leading to the increase in $R_{\max }$.

Noteworthy is that the semisaturation contrast increased significantly during cooling by $35 \%$ (median; $p<0.0001$ ). Together, the dominating influences of interhemispheric input we observed were changes in responsiveness (response gain) and sensitivity (contrast gain) to stimulus contrast. To explore the relationship between these two parameters, we applied a com- bined fit to the data, keeping the parameters $s$ and $n$ constant between warm and cool states, leaving $R_{\max }$ and $C_{50}$ free to change (Eqs. 2, 3; see Materials and Methods). We calculated a modulation index for these two parameters (Eq. 4), with negative values indicating a decrease during cooling compared to the warm state and positive values indicating an increase. In Figure $2 C$, these indices are plotted against each other together with their 95\% confidence intervals. Most points are located in the lower right quadrant, indicating a decrease in $R_{\max }$ and an increase in $C_{50}$ during deactivation. However, the correlation between both modulation indices is positive (Spearman $\rho=0.35, p<0.0001$ ). This is because data points with strong positive effects for one parameter tend to lie around zero for the other parameter, indicating that neurons with a strong change in $R_{\max }$ tend to have a small change in $C_{50}$ and vice versa.

Contribution of contrast and response gain to scaling of CRFs Given the results above, it seems unlikely that the effect of interhemispheric input can be described as a pure response or contrast gain. We therefore fit a "full model" including both $R_{\max }$ and $C_{50}$ as free parameters and compared the quality of this fit to that of the two "reduced models," with either $R_{\max }$ or $C_{50}$ being a free parameter between warm and cooling states (Eq. 5). Figure $3 A$ shows this procedure for three multiunits, which represent the different types of modulation we observed throughout our sample. For all three multiunits, the full model provides an excellent fit, indicated by adjusted $R^{2}$ values of $0.996,0.987$, and 0.983 , respectively. For Unit 1, the response gain model failed to adequately describe the CRFs during the warm (black line) and cooling (gray line) states. Nevertheless, the variance explained by the model was still very high $\left(R^{2}=0.964\right)$. This is because the hyperbolic ratio function has the general form of a typical CRF. We therefore expressed the goodness of fit of the reduced models as their performance relative to the full model (reduced model per- 
A
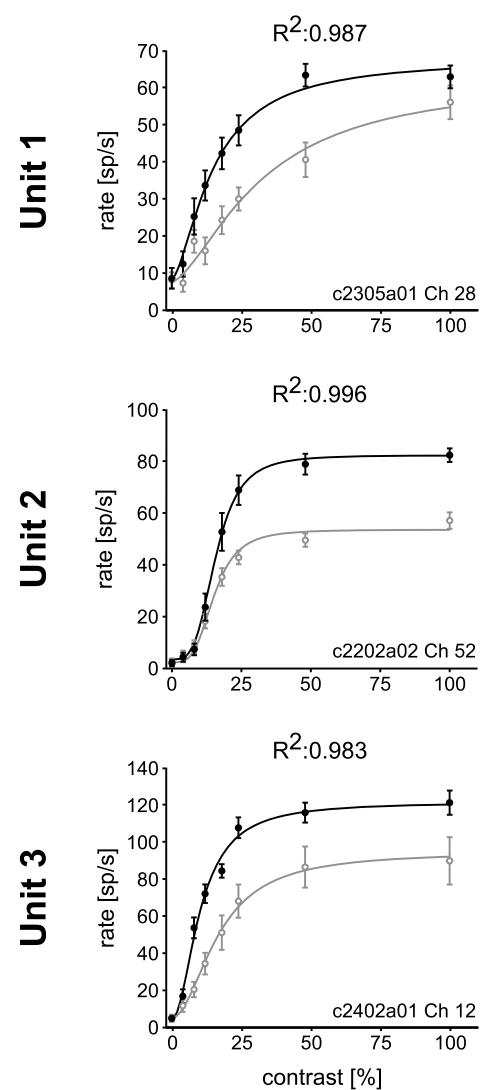

\section{Contrast-Gain}

Model
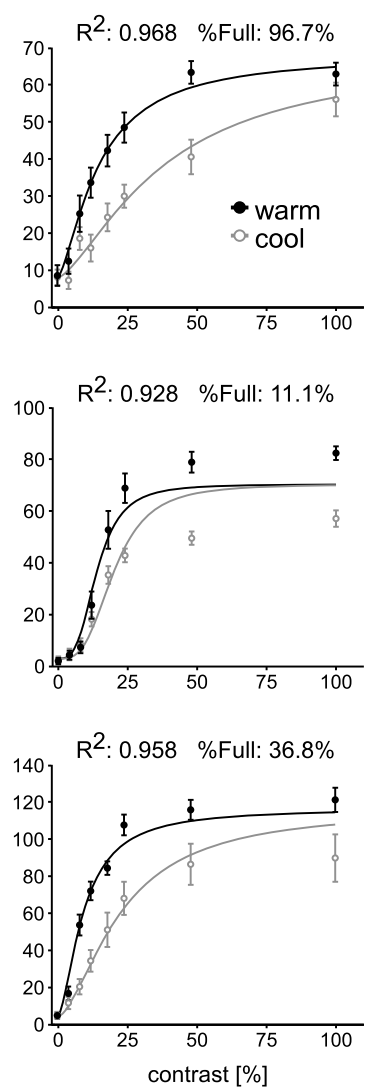

\section{Response-Gain Model}
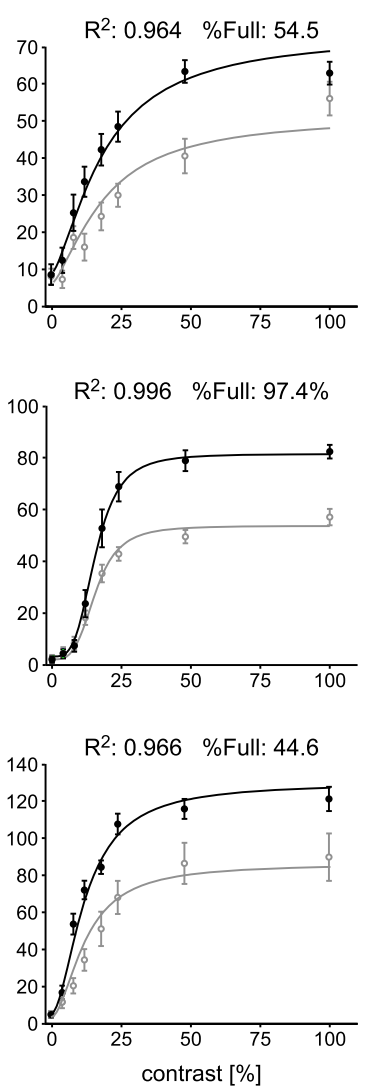

C

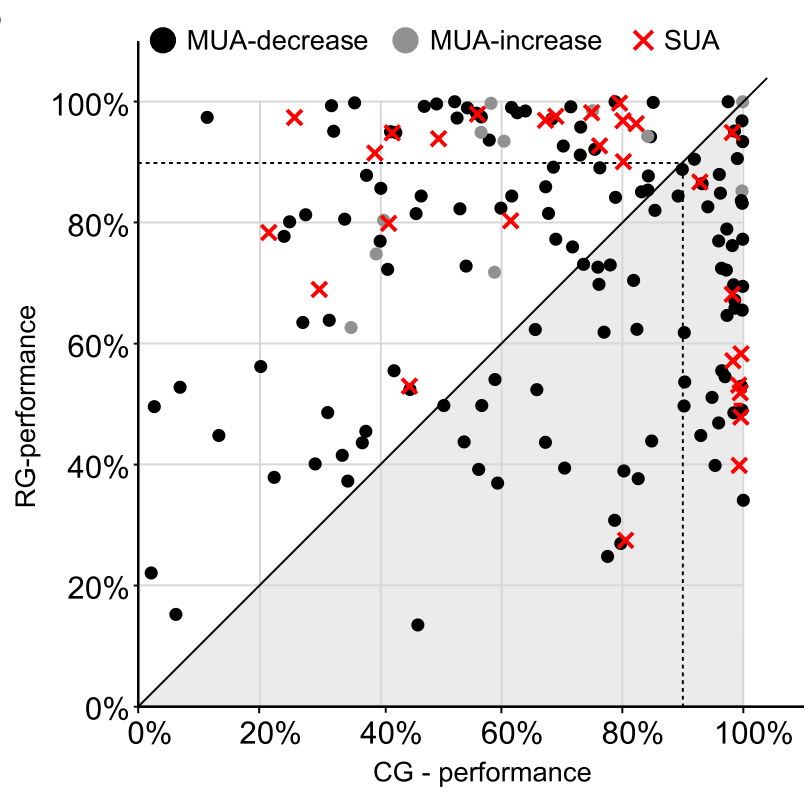

preferred orientation

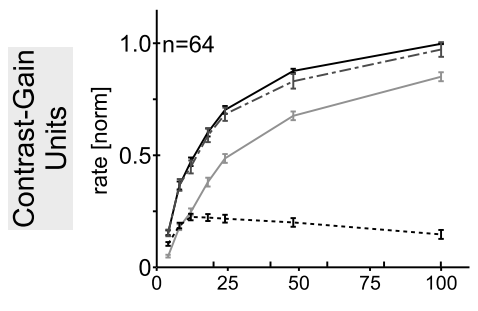

- warm - cool -.-rewarm ..... warm-cool
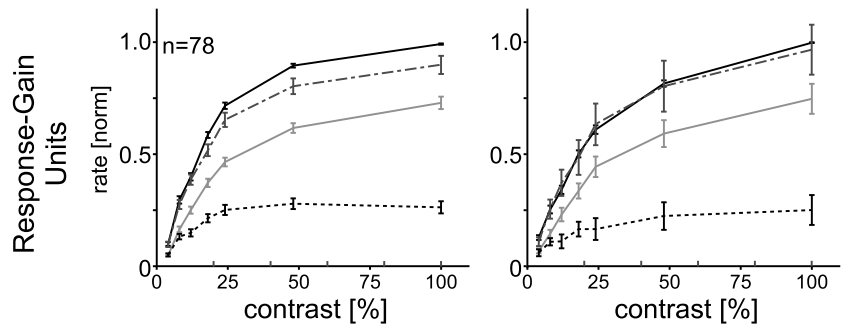

Figure 3. A, Example of three multiunits being modulated by a contrast gain (top row), response gain (middle row), or combination of both (bottom row). $\boldsymbol{B}$, Model performance for all recording sites; $100 \%$ means a contrast or response gain model is as good in explaining the data as the full model. Black and gray dots indicate multiunits with average rate decreases and increases, respectively, and red crosses indicate single units obtained by spike sorting. Data points below the diagonal (gray shading) are classified as contrast gain multiunits, and those above as response gain multiunits. C, Average normalized contrast response functions in the warm (black), cooled (gray), and rewarmed (dashed) states for the contrast and response gain multiunits, shown separately. The dotted lines indicate the difference between the warm and cool states. Data are given for the preferred orientation of each unit and $45^{\circ}$ away from the preferred orientation. 
formance, Eq. 6). This fraction was only $55 \%$ for the example unit presented in Figure $3 A$. In contrast, the contrast gain model provided an excellent fit for this unit, with an RMP of $96.7 \%$. For Unit 2, the opposite was true, with the response gain model providing a better fit (RMP, 97.4\%) than the contrast gain model (RMP, 11.1\%). Interestingly, Unit 3 could only be described by a combination of contrast and response gain. The performance of each model alone was only a fraction of the full model $(36.8 \%$ and $44.6 \%$ for the contrast and response gain models, respectively).

In Figure 3B, the performance of the contrast gain model is compared with that for the response gain model for all multiunits. It becomes clear that across all multiunits we can observe a continuum between those that can be described by one of the gain models alone and those that can only be adequately described using both a contrast gain and a response gain parameter (Fig. 3A, Unit 3). One possibility for the latter group is that these multiunits are noisier, and thus provide a worse fit. We therefore performed a median split on the model performance for contrast and response gain, sorting the multiunits in two groups: those that could be fit well by the contrast or response gain model (or both) and those that could not (Fig. 3B, median border marked by dashed lines). Interestingly, those multiunits that could not be fit well with the reduced models were fit even better by the full model (average $R^{2}=0.983$ ) than those multiunits well described by either of the reduced models (average $R^{2}=0.970$ ). This indicates that the multiunits with a low model performance for contrast or response gain alone indeed were not noisier, but could only be adequately described by including both parameters, $R_{\max }$ and $C_{50}$, into the model. We separately plotted single units obtained by spike sorting (Fig. 3B, red crosses). There is a clear tendency for the single units to prefer one or the other gain mechanism. Eighty percent of the single units had a model performance of $>90 \%$ for the contrast or response gain mechanism. This indicates that a given neuron is usually modulated by an input or output gain modulation, but can be occasionally influenced by both mechanisms simultaneously.

For further analysis, we put the multiunits into two groups to simplify: those that could be better described by a contrast gain mechanism (Fig. 3B, data points below the diagonal) and those better described by a response gain (Fig. $3 B$, data points above the diagonal). This allowed us to replot the data with respect to the scaling mechanism that best described the neurons' behavior. Figure $3 C$ (left) shows the average normalized CRFs for contrast $(n=64)$ and response gain $(n=78)$ multiunits at their preferred orientation after subtracting spontaneous firing. As expected from the scatter plot (Fig. 3B), the curves do not follow a perfect contrast or response gain (compare Figs. 1, Fig. 3B), but the dominant mechanism is clearly visible (i.e., from the difference between the warm and cool conditions indicated by the dotted line). The average effect was a significant decrease of firing rate at all contrasts $(t$ test, $p<0.007)$ for both groups. However, the scaling of a CRF by a response gain mechanism could potentially look like a contrast gain, because of a ceiling effect at high contrasts; that is, the interhemispheric input may not be able to increase the firing of the neurons at high contrast, because they already fire at maximum. We therefore analyzed the CRFs at the orientation $45^{\circ}$ away from each unit's preferred orientation. As shown in Figure $3 \mathrm{C}$, the contrast gain multiunits still show a shift along the contrast axis. All effects were reversible, indicated by a recovery to baseline after rewarming.

\section{Contrast dependence of orientation tuning}

We showed above that interhemispheric input scales the CRF of a particular neuron with a bias toward either a contrast or a response gain. Therefore, the question arises of whether those neurons are also differently influenced with regard to their orientation tuning. As mentioned in the introduction, the interhemispheric input was shown previously to scale the responses of orientation tuning curves in a multiplicative manner, although some additive affects were also observed. Here, we present the average orientation tuning curves for different contrasts in the warm (black lines) and cooling (gray lines) states separately for those multiunits classified as contrast and response gain (Fig. $4 A$ ). Individual tuning curves were normalized to peak responses during the warm state, after subtracting spontaneous activity. As expected, the effect of cooling was stronger at lower contrasts for the contrast gain multiunits. However, a perfect contrast gain mechanism would predict the maximal change at intermediate contrasts, with no effect at the lowest ones. This is clearly not the case for our data, where the strongest (relative) effects are present at the lowest contrasts. The decrease in absolute firing rate, however, was weaker for the two lowest contrasts (4 and 8\%) than for the intermediate contrast (12\%). For the response gain multiunits, the relative decrease in activity was fairly uniform across contrasts, as expected for this scaling mechanism. Most importantly, the scaling of responses for each orientation tuning curve resembles a multiplicative change at each contrast for both the contrast and response gain multiunits. To explore this in detail, we fit a Gaussian model to each tuning curve in the warm and cool state (Eq. 8). Similar to CRFs, we expressed a change in the respective parameter between the states by a MI, with a negative value representing a decrease of the parameter during cooling. The results for each parameter are shown in Figure $4 B$ for the contrast gain (left) and response gain (right) multiunits. For each parameter, we tested for differences in the MI across contrasts by a repeated-measures (RM)-ANOVA. Individual changes at each contrast were assessed with a nonparametric permutation test (simultaneous across all contrasts), controlling for multiple comparisons ( $n=10,000$ resamplings). For the contrast gain multiunits, the amplitude parameter of the fitted Gaussian function showed a strong dependence on contrast, as expected from the plots above (RM-ANOVA, $p<0.0001$ ). The decrease was significant $(p<0.001)$ for each contrast except the highest $(p=0.09)$. The offset, representing the DC component of the tuning curve, was significantly reduced at $4 \%$ and $12 \%$ contrast $(p<0.05)$, but there was no significant difference between contrasts as assessed by RM-ANOVA. The tuning width, expressed as the SD of the tuning curve, as well as the preferred orientation, did not change during cooling (RM-ANOVA and individual permutation tests, both $p>$ $0.1)$. For the response gain multiunits, the results were similar. However, the only significant effect was a decrease of the tuning amplitude at each contrast (permutation test, $p<0.05$ ). This decrease was different between contrasts (RM-ANOVA, $p<0.0001$ ); however, it was not as pronounced as for the contrast gain multiunits.

The present analysis shows that the effect of interhemispheric input on the orientation tuning curves is a change in their amplitude (and for some contrasts in their offset), without a change in tuning selectivity or preferred orientation, in agreement with a multiplicative gain change for both contrast and response gain multiunits. 

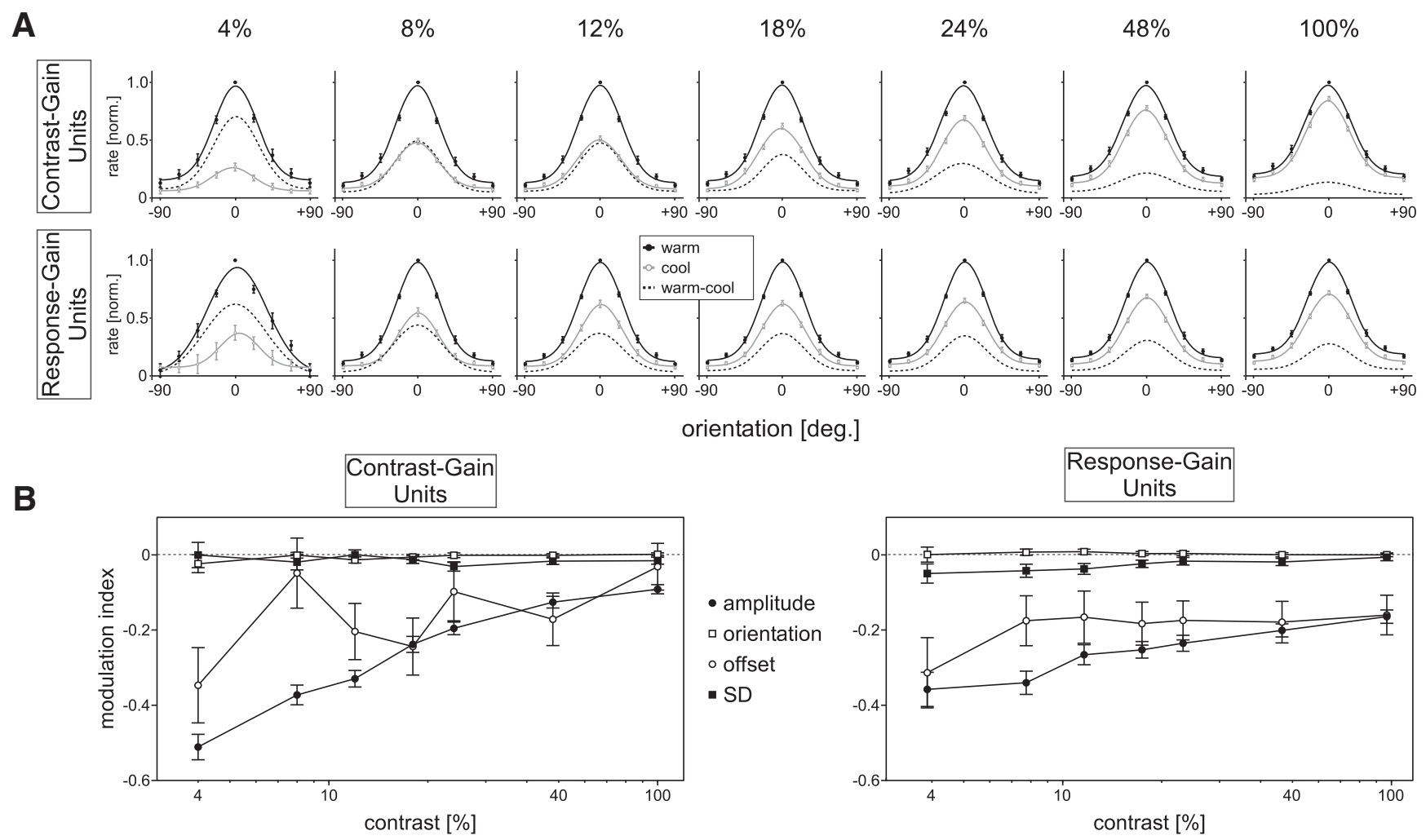

$48 \%$

$100 \%$

Figure 4. Analysis of orientation tuning curves. $\boldsymbol{A}$, Average normalized orientation tuning curves at each stimulation contrast for the contrast and response gain multiunits. The dotted lines indicate the difference between the warm and cool states. For both groups, firing rates are scaled approximately multiplicatively at each contrast. $\boldsymbol{B}$, Change in the parameters of a Gaussian fit to individual tuning curves for the contrast gain (left) and response gain multiunits (right). A negative modulation index indicates a decrease of the parameter during cooling. Significant changes are only present for the amplitude and the DC offset of the tuning curve.

\section{Dependence of scaling mechanism on cellular properties}

In the previous paragraphs we discussed that the effect of interhemispheric input can be described as either a contrast or a response gain. Here, we ask whether a specific property of the neurons determines the scaling mechanism. We tested four classes of properties: (1) RF, (2) tuning, (3) CRF, and (4) laminar position. All properties were obtained in the warm state. To be able to correlate the type of scaling mechanism with the various neuronal properties, we calculated a GI, indicating how well a given change in firing rate can be described by either the contrast or the response gain mechanism (Eq. 7). A GI of $<0$ indicates a contrast gain mechanism, whereas a GI $>0$ represents response gain.

\section{Receptive field properties}

We obtained receptive field position (azimuth and elevation) and size by an automatic mapping procedure described in Materials and Methods. The RF positions given here are relative values, normalized within each animal. Given our recording sites, absolute RF positions were usually close to the vertical and horizontal meridian. We also classified the recorded neurons into simple and complex, based on their response modulation to the drifting gratings (for details, see Materials and Methods). In Figure $5 \mathrm{~A}$ (top row), the RF properties are plotted against the gain index. We used the nonparametric Spearman rank correlation to determine whether there was a relationship between the two. The $95 \%$ confidence interval (obtained by a bootstrap test, $n=1000$ resamplings) is indicated together with the correlation coefficient above each plot. We detected a weak but significant positive correlation between GI and RF size, indicating that bigger RFs tend to benefit from a stronger response gain contributed by callosal input.

\section{CRF properties}

It could be that a specific CRF shape itself dictates the scaling mechanism. We therefore correlated GI with the four parameters of the hyperbolic ratio functions (Fig. $5 A$, middle row). There was a strong relationship of the shape parameter $(n)$ with the scaling mechanism $(r=0.51)$, indicating a response gain change for those multiunits with a steep CRF slope. Correlation with the other parameters was weak or not significant.

\section{Tuning properties}

We also tested for a correlation between GI and the direction/ orientation selectivity (expressed as a DSI or OSI) and the preferred direction/orientation of the neuron (Fig. 5A, bottom row). There was a weak but significant correlation between GI and DSI, indicating a stronger response gain for neurons with higher direction selectivity.

\section{Laminar position}

To examine whether contrast and response gain multiunits distributed differently across cortical layers, we recorded from laminar probes in area 17 close to the vertical meridian in one animal (Fig. 5B). We inserted the probes during visual inspection until the uppermost contact vanished in the brain tissue and started to record action potentials. According to the probe's length (16 contacts with a $100 \mu \mathrm{m}$ intercontact spacing), we assumed the middle (granular) layer 4 to be recorded on the center contacts. This was confirmed by a current source density (CSD) analysis (sec- 
A
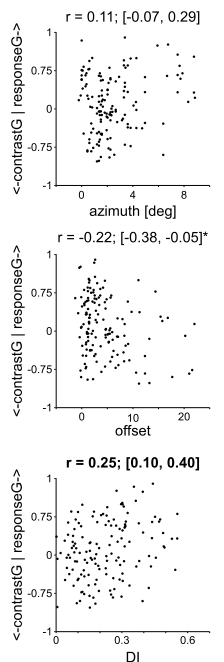
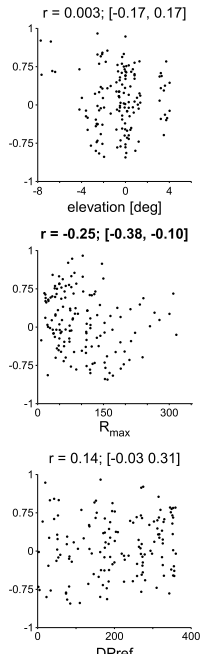
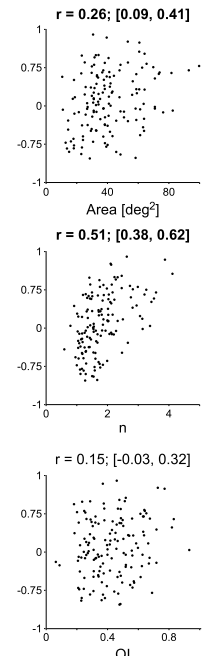
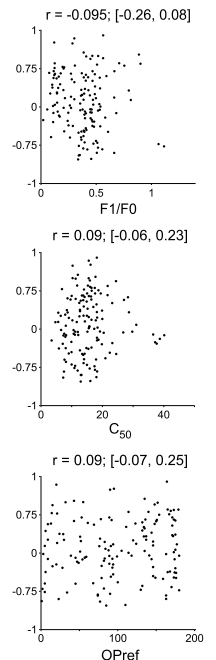

B

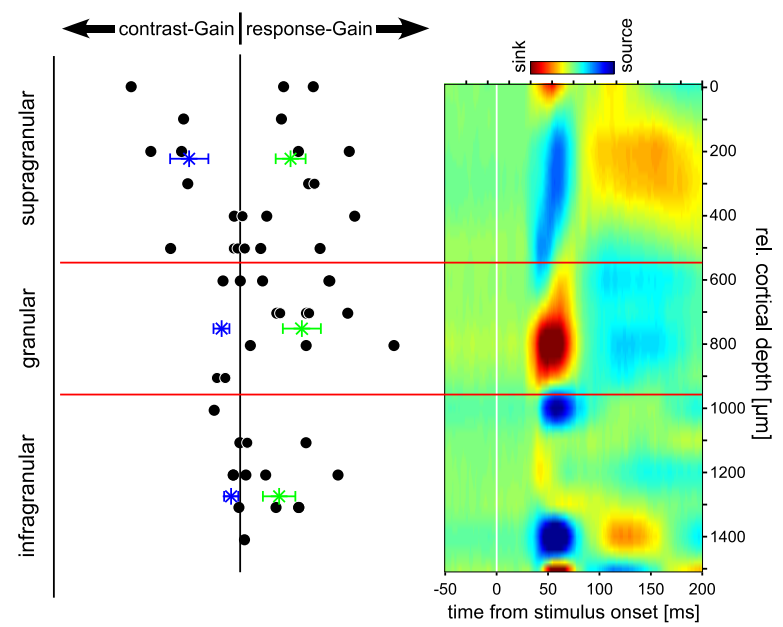

Figure 5. Dependence of scaling mechanism on cellular properties. $\boldsymbol{A}$, Various cellular properties are plotted as a function of the scaling mechanism (expressed as a gain index; for details, see Materials and Methods). The Spearman rank correlation coefficient and the $95 \%$ confidence interval are given above each plot (significant correlations are indicated in bold). $\boldsymbol{B}$, Laminar analysis of the scaling mechanism for the data obtained from one cat. Depth of recording was verified by a CSD analysis (right). The black dots (left) label individual multiunits, and stars indicate the mean for each compartment. The contrast gain mechanism is most prominent in the supragranular layers, whereas a response gain exists throughout all layers.

ond spatial derivative across channels followed by interpolation; Mitzdorf and Singer, 1979) of local field potentials recorded simultaneously with the multiunit activity. In the CSD analysis, the first current sink after stimulus onset indicated the input layer 4 .

By separating contrast and response gain multiunits, we found that contrast gain was stronger in the supragranular than in the granular or infragranular layers $(p<0.05$, ANOVA). Interestingly, the supragranular layers are those where direct callosal input is to be most expected. For the response gain mechanism, there was no significant difference between layers. Thus, contrast and response gain cells distributed significantly differently among layers. Since both electrodes sampled both upper and lower layers, a sampling bias in depth seems to be unlikely to have caused the significant ANOVA test. A spatial sampling bias would be relevant if there existed scattered infragranular islands of contrast gain units, which we accidentally did not hit. In that case, we would erroneously assume an anisotropic distribution of contrast and response gain units. However, according to the hypothesis that contrast gain units predominantly receive direct callosal input, it is rather likely that we underestimated the number of supragranular contrast gain units, as there exist acallosal bridges in area 18 in carnivores (Manger et al., 2002).

Given the dependence of the scaling mechanism on some of the properties tested, especially the strong dependence on the steepness of the CRF, these findings indicate that the network a particular neuron is embedded in biases the scaling mechanism toward a contrast or response gain mechanism.

\section{Model of interhemispheric impact}

So far, we have shown that interhemispheric input can affect neurons by a contrast gain mechanism, a response gain mechanism, or a combination of both. Furthermore, it seems that responses to different orientations are scaled multiplicatively, with the strength determined by the stimulus contrast and gain mechanism. We also found that the mechanism present is correlated to the steepness of the CRF, a parameter most likely determined by the connectivity of a particular neuron. Based on these observations, we developed a simple model describing the effect of inter- hemispheric input on the CRF and orientation tuning curves. This model is not meant to capture the precise biophysical properties of synaptic integration, but to provide insight into the putative components of the underlying mechanism. In most models of gain modulation, the modulating input is a constant with regard to the stimulus parameters (Murphy and Miller, 2003; Ayaz and Chance, 2009). This is obviously not the case in our experimental setup. As we presented stimuli in both visual hemifields, the incoming callosal input is also dependent on the stimulus. The model is constrained in some parameters based on the anatomy and physiology of interhemispheric connections. Among them, connections are assumed to be excitatory (Conti and Manzoni, 1994) and link neurons with similar responses to contrast (Berardi et al., 1987; Tardif et al., 1997) and orientation (Schmidt et al., 1997; Rochefort et al., 2009). Furthermore, the CRF is assumed to scale the amplitude of the orientation tuning curves, keeping tuning selectivity constant across contrasts (Sclar and Freeman, 1982).

A sketch of the model is presented in Figure $6 A$. The neuron surrounded by the dashed circle represents the recorded neurons. It receives three types of synaptic input, as follows.

The first type of input is noncallosal input (NCI; Fig. 6, black), which can originate from direct geniculocortical afferents or from other neurons in the same or a different layer. The inputs are a combination of the two vectors for stimulus contrast (SC) and stimulus orientation (SO):

$$
\operatorname{NCI}(c, \theta)=\operatorname{SC}_{\text {dir }}(c) \times \operatorname{SOT}_{\text {dir }}(\theta)
$$

where SC is modulated in the contrast dimension and constant in orientation, and vice versa for SO. The $\mathrm{T}$ in the equation above indicates vector transposition. Stimulus contrast is modeled as a hyperbolic ratio function:

$$
\operatorname{SC}_{\operatorname{dir}}(c)=\frac{c^{n}}{c^{n}+c_{50}^{n}}
$$




\section{A}

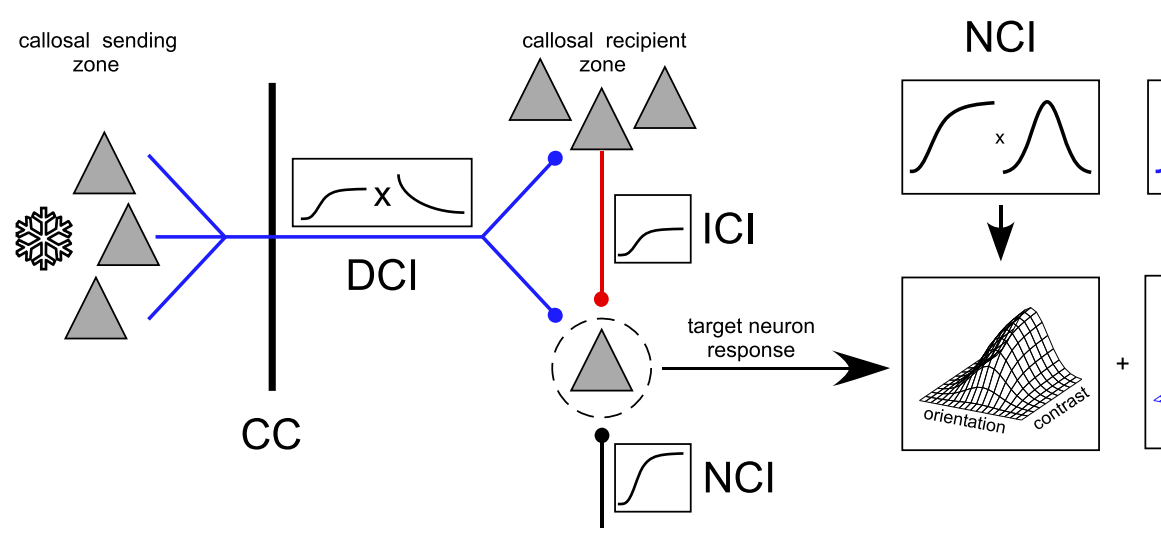

$\mathrm{DCl}$

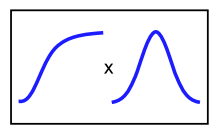

$\downarrow$

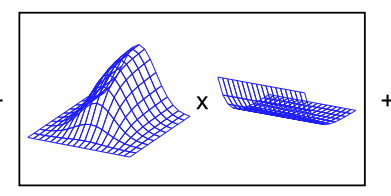

$\mathrm{ICl}$

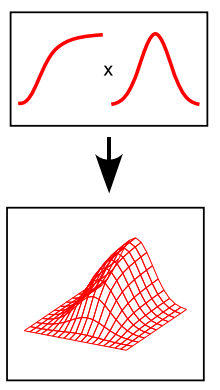

C
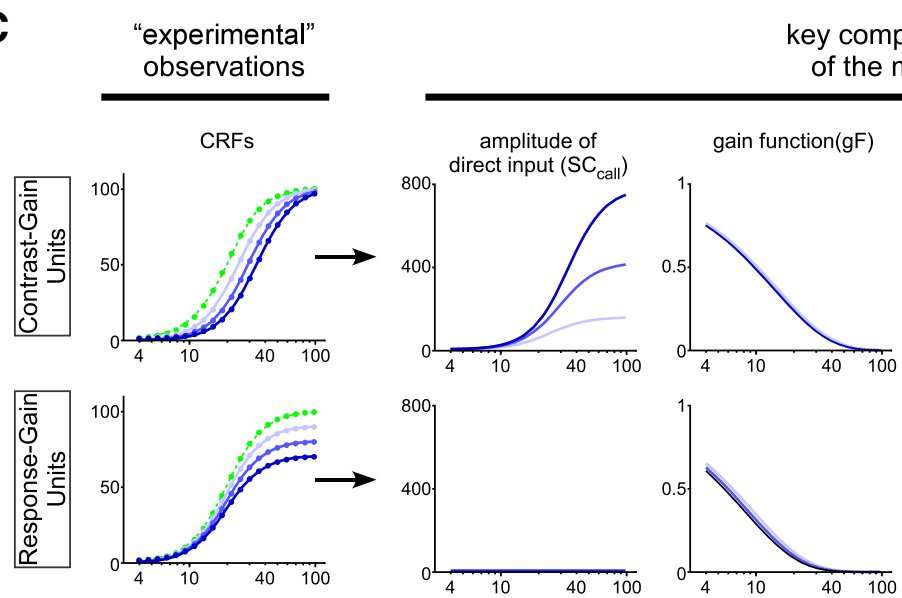

key components of the model
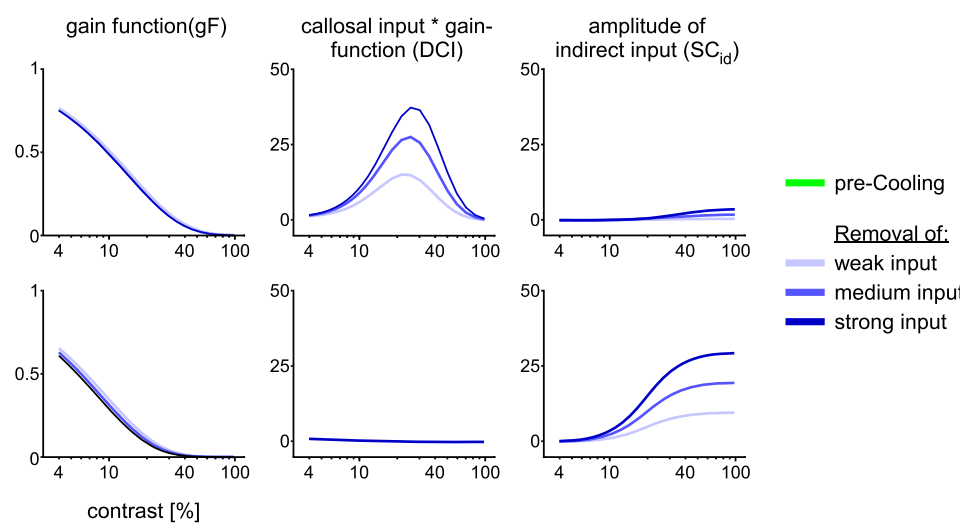

Figure 6. Model of interhemispheric impact on CRFs. $A$, Sketch of the model describing the influence of interhemispheric input. A given neuron (inside dashed circle) is supposed to receive three types of input: noncallosal input, direct callosal input, and indirect callosal input. $\boldsymbol{B}$, Activity was modeled as a combination of contrast and orientation. In the case of DCl, the activity was weighted by a contrast-dependent gain function. C, Model behavior for a pure contrast gain (top row) or response gain (bottom row) mechanism. Starting from the green curve (intact interhemispheric input), the activity was shifted to the right for the contrast gain mechanism and downward for the response gain mechanism (removing different levels of input). The behavior of the model components is coded in the same colors as the shifts of the (RFs. The unit of the $y$-axis is input rate for all plots except the gain function, which was set to range between 0 and 1.

$c$ is stimulus contrast, $n$ the steepness of the function, and $C_{50}$ the semisaturation contrast. Orientation tuning is modeled using a Gaussian function:

$$
\mathrm{SO}_{\mathrm{dir}}(\theta)=A_{\mathrm{dir}} \frac{-\theta-\theta_{p}^{2}}{2 \sigma^{2}}+B_{\mathrm{dir}},
$$

where $\theta$ is orientation, $A_{\mathrm{dir}}$ is the amplitude of the direct input, $\theta_{\mathrm{p}}$ is the peak orientation, $\sigma$ is the $\mathrm{SD}$, and $B_{\text {dir }}$ is a baseline parameter representing the DC offset of the direct input. The multiplication of the two curves is shown as a $3 \mathrm{D}$ mesh plot in Figure $6 B$.

The second type of input is direct callosal input (DCI; Fig. 6, blue). The DCI is modeled in a similar way as the NCI, sharing the parameters $n, C_{50}, \theta_{\mathrm{p}}$ and $\sigma$, having the amplitude $\left(A_{\text {call }}\right)$ and baseline $\left(B_{\text {call }}\right)$ as free parameters. However, in our model, the $\mathrm{NCI}$ is scaled by a contrast-dependent gain function $(\mathrm{gF})$. Such a function divides the input for increasing stimulus drive, namely, contrast, as proposed by the divisive normalization model (Carandini and Heeger, 2012) and observed experimentally (Cardin et al., 2008). We assumed this gain function to be a decreasing exponential:

$$
\mathrm{gF}(c)=\exp \left(-\frac{c}{\tau}\right)
$$

with $\tau$ is determining how rapid the function decreases to 0 . The gain function is constant across orientations. The direct callosal input as a function of contrast and orientation is therefore modeled as follows:

$$
\operatorname{DCI}(c, \theta)=\left[\mathrm{SC}_{\text {call }}(c) \times \operatorname{SOT}_{\text {call }}(\theta)\right] \times \mathrm{gF}(c, \text { const. }) .
$$

The third type of input is indirect callosal input (ICI; Fig. 6, red). ICI is also modeled as a combination of stimulus contrast and stimulus orientation. It represents an indirect effect of callosal input, which is not weighted by a contrast-dependent gain function, and may originate from neurons nearby themselves receiving direct callosal input. The parameters $n, C_{50}, \theta_{\mathrm{p}}$ and $\sigma$ are the same as for the NCI and DCI; amplitude $\left(A_{\mathrm{id}}\right)$ and baseline $\left(B_{\mathrm{id}}\right)$ are free parameters:

$$
\mathrm{ICI}(c, \theta)=\operatorname{SC}_{\mathrm{id}}(c) \times \operatorname{SOT}_{\mathrm{id}}(\theta) .
$$


We performed a simultaneous fit to the data obtained during warm and cool states. A constrained nonlinear optimization algorithm was used to obtain the fit parameters ("trust-regionreflective" implemented in the Matlab function "Isqnonlin"). More specifically, the data for the cool state were assumed to receive only noncallosal input, because cooling blocks the DCI and ICI contributions. We therefore minimized the RSS between the data during cooling and the modeled NCI:

$$
\min \operatorname{RSS}_{\text {cool }}=\min \sum_{c, \theta}\left\{\operatorname{Data}_{\text {cool }}(c, \theta)-\mathrm{NCI}(c, \theta)\right\}^{2} .
$$

A neuron is assumed to receive all three types of inputs during the warm state, but the relative contribution is weighted by the value of the respective amplitude parameter. We therefore minimized the sum of squares between the data in the warm state and the sum of all synaptic inputs:

$$
\begin{aligned}
\min \operatorname{RSS}_{\text {warm }}= & \min \sum_{c, \theta}\left\{\operatorname{Data}_{\text {warm }}(c, \theta)\right. \\
& -[\operatorname{NCI}(c, \theta)+\operatorname{DCI}(c, \theta)+\operatorname{ICI}(c, \theta)]\}^{2} .
\end{aligned}
$$

To obtain the fit parameters, we minimized the combined sum of squares for cool and warm. We constrained some of the parameters to avoid biologically implausible parameter values. The upper and lower bounds were as follows: $A_{\mathrm{dir}}\left[0, A_{\text {cool }}{ }^{*} 2\right], \theta_{\mathrm{p}}[5,90]$, $\sigma[0,360], B_{\text {dir }}\left[0, B_{\text {cool }}\right], n[0, \operatorname{Inf}], C_{50}[0$, Inf $], A_{\text {cal }}[0$, Inf $], B_{\text {cal }}$ $[0, \operatorname{Inf}], A_{\text {id }}\left[-A_{\text {warm }}, A_{\text {warm }}\right], B_{\text {id }}\left[0, B_{\text {warm }}\right], \tau[0$, Inf $]$. The negative amplitude for the indirect input was chosen to allow for inhibitory effects.

We first applied the model to synthetic data consisting of a CRF scaled only by its $C_{50}$ (contrast gain multiunits; Fig. $6 C$, top row) or $R_{\max }$ (response gain multiunits; bottom row). We specifically asked the model how the individual parameters change given a certain cooling-induced shift in the CRF. A progressive increase in $C_{50}$ ( $5 \%$ contrast steps) led to an increase of the direct callosal input. The gain function did not change between states, with a $\tau$ of $\sim 14 \%$ contrast. However, the multiplication of the gain function with the direct callosal input resulted in a function having its maximum at an intermediate contrast $(26 \%)$. The contrast-dependent gain function therefore transformed the sigmoidal shape of the callosal input into a new function, leading to a rightward shift when added to the receiving CRF. The contribution of the indirect input was only marginal for the contrast gain mechanism. A progressive change in $R_{\max }$ (10 spikes/s steps), on the other hand, gave a different result. Here, the direct callosal input was near zero, whereas the indirect input increased progressively. The addition of the indirect input to the receiving CRF (with the same shape parameters) led to a pure change in $R_{\max }$ corresponding to the multiplicative scaling by a constant factor. Those results indicate that a neuron can change its CRF by a contrast or response gain mechanism, depending on the relative contribution of direct and indirect callosal input. Direct callosal input seems to contribute more to a contrast gain mechanism, whereas indirect callosal input is likely to be involved in the response gain mechanism. This supports our interpretation of Figure $5 B$ that the prevalence of contrast gain in supragranular layers reflects direct callosal input.

Next, we applied the model to the 142 multiunits recorded. Figure $7 A$ shows box plots of the parameter values, split by those units determined previously to be contrast gain or response gain multiunits. To facilitate comparison between direct and indirect parameters, we $z$-scored $R_{\max }$ and $C_{50}$. The other parameters are in their respective units. As expected, for the contrast gain multiunits, $R_{\max }$ was larger for the direct input than for the indirect one (Mann-Whitney $U$ test, $p<0.01$ ). Correspondingly, for the response gain multiunits, $R_{\max }$ was larger for the indirect input $(p<0.001)$. This was also true when comparing contrast and response gain within one input type. The offset for the direct input was (in original units) close to zero. For the indirect input, some multiunits required a particular offset, representing a DC shift. The tuning width (SD) and center of the orientation component, as well as the slope of the gain function $(\tau)$, did not differ significantly between contrast and response gain. Interestingly, the slope parameter $(n)$ was larger for the response gain multiunits than for the contrast gain ones, as shown in Figure 5A. Also, the semisaturation contrast $\left(C_{50}\right)$ was larger for the contrast gain multiunits. This is surprising because we did not find a correlation between $C_{50}$ and the scaling mechanism in the previous analysis.

Next (Fig. 7B), we averaged the individual model components (inputs), as for the synthetic data shown in Figure 6C. Once more, it becomes obvious that for contrast gain multiunits, the direct callosal input dominates over the indirect input and vice versa for the response gain multiunits.

To draw predictions from our model, we had to make sure that it fit well to the data. Therefore, we tested the goodness of fit by evaluating the $R^{2}$ value across orientations (at highest contrast; Fig. $7 C$, top) and contrast (at preferred orientation; Fig. $7 C$, bottom). Generally, the model provided a good fit for both contrast and response gain multiunits, deteriorating for nonpreferred orientations and low contrast. As an additional control, we performed a bootstrap analysis ( $n=1000$ resamplings) to get an estimation of the variability of responses for the real data and the model. Figure $7 D$ plots the average normalized CRFs and tuning curves (points) together with the model (lines). The confidence intervals for data and model largely overlap, indicating a good fit. For the tuning curve at low contrast, however, one observes some slight deviations, in accordance with the reduced R2 value described previously. In summary, the present model proposes a cell-specific contribution of different input streams, whose relative contribution determines the scaling mechanism.

\section{Discussion}

In the present study, we investigated how different signal streams are combined in the primary visual cortex. We reversibly deactivated a restricted part of the cortex to control the synaptic input to a spatially distinct neuronal population. It turned out that this synaptic input could change the overall output (response) gain of the target neuronal population. However, we also found clear evidence for an input (contrast) gain modulation. Importantly, orientation tuning was scaled multiplicatively for all stimulus contrasts; i.e., we found a response gain in the orientation domain. This implies that the action of the lateral interhemispheric network distinguishes between stimulus features, being sensitive to contrast but invariant to orientation.

\section{Input and output gain modulation through the interhemispheric network}

Most of the neurons we recorded decreased their firing rate upon thermal deactivation of the contralateral hemisphere. This is in line with previous reports (Payne et al., 1991; Sun et al., 1994; Schmidt et al., 2010; Peiker et al., 2013; Wunderle et al., 2013) and the predominant excitatory nature of the interhemispheric connections (for review, see Conti and Manzoni, 1994). The rate decreases we observed are surprisingly strong, given the small 
A

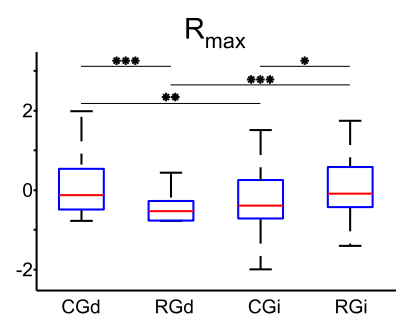

Center

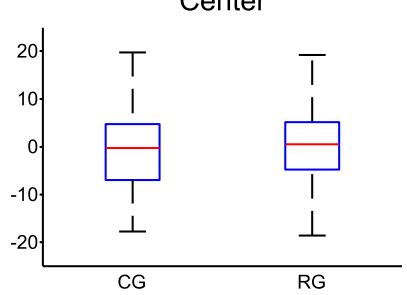

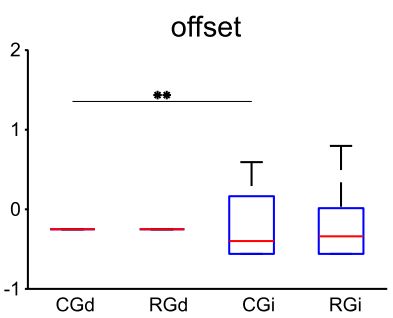

$\mathrm{n}$

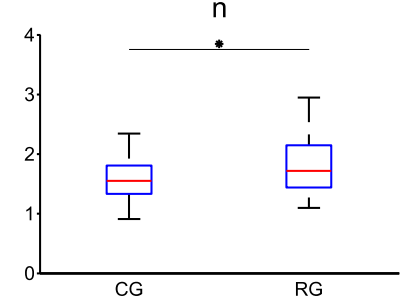

SD

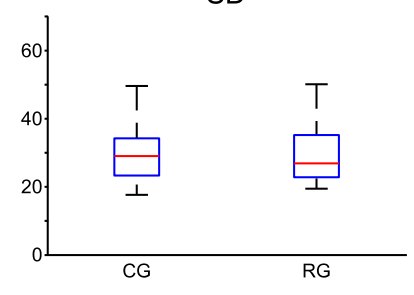

c50

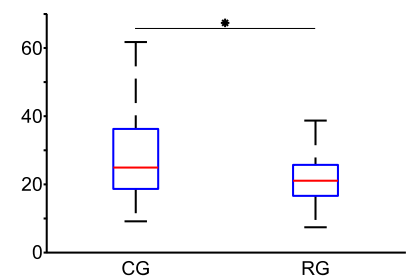

$\tau$

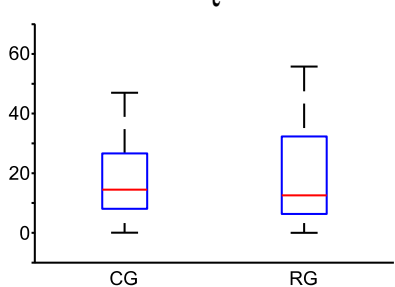

B
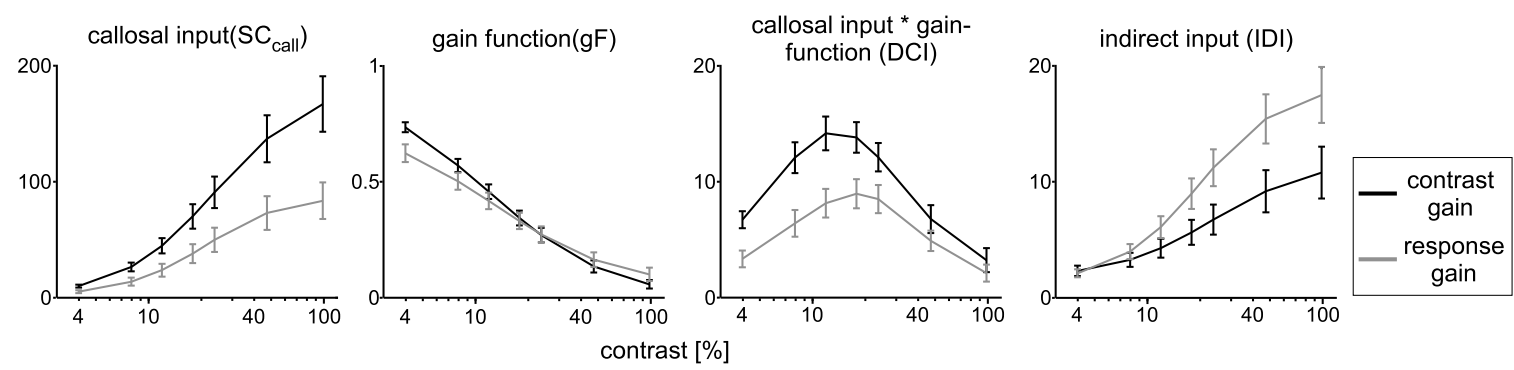

C
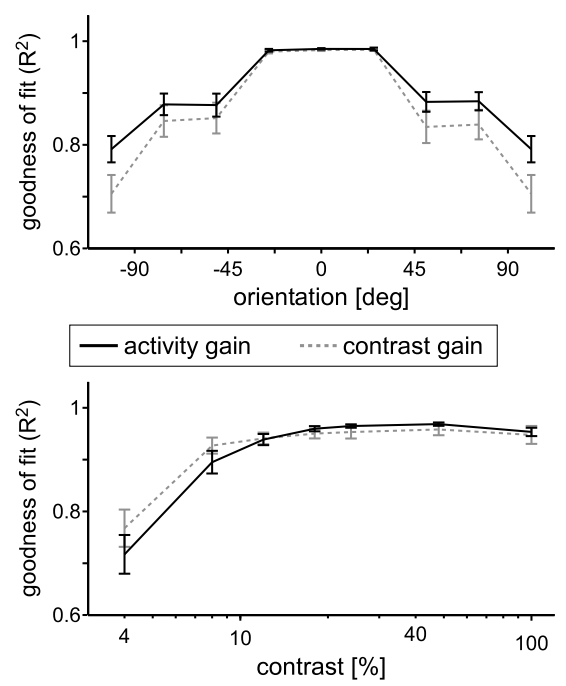

D
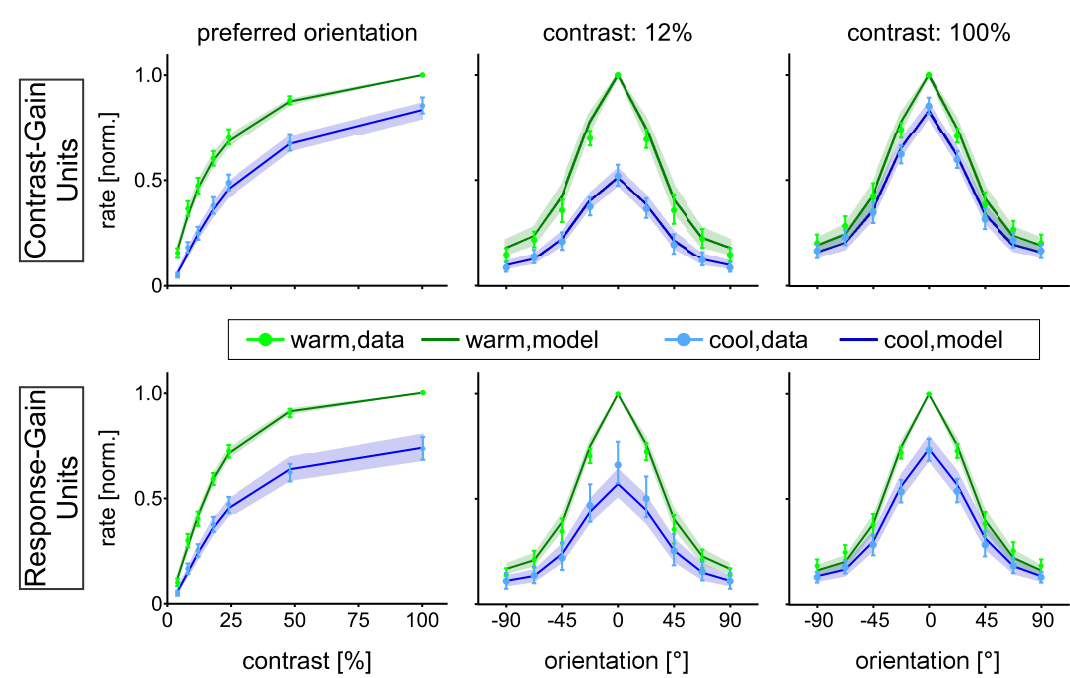

Figure 7. Performance of the callosal model. The model was fitted to each recorded multiunit individually. $\boldsymbol{A}$, Box plots of the model parameters describing the callosal influence for those multiunits classified as contrast or response gain. The parameters $R_{\max }$ and offset were normalized for comparison between contrast and response gain. $\boldsymbol{B}$, Average fitted model components are as in Figure 6 C. The lines indicate the mean for contrast (black) and response gain multiunits (gray). C, Goodness of fit across orientations for the highest contrast (top) and across contrasts for the preferred orientation (bottom). D, Average normalized contrast response function and orientation tuning curves (for a low and high contrast) separate for the contrast and response gain multiunits. The mean and $95 \% \mathrm{Cl}$ for the data are given by the dots and error bars; the mean and $95 \% \mathrm{Cl}$ for the model are given by the lines and transparent shadings. CG, Contrast gain; RG, response gain. ${ }^{*} p<$ $0.05 ; * * 0.01 ; * * * 0.001$.

fraction of callosal synapses on a typical dendritic tree (Porter and White, 1986). However, this could be explained by a local amplification of the incoming interhemispheric input through recurrent connections (Douglas et al., 1995), as was proposed previously for the intrinsic horizontal network (Reynaud et al., 2012).
Previously (Wunderle et al., 2013) we demonstrated that the interhemispheric input leads to a multiplicative scaling of orientation tuning curves. Such a response gain mechanism describes a rather unspecific increase or decrease in the output response of a neuron or a population of neurons. As a consequence, those neurons can modulate their level of activity without changing their 
tuning properties. For example, orientation tuning is known to be invariant with stimulus contrast, keeping tuning selectivity despite changes in overall stimulus drive (Sclar and Freeman, 1982). By analyzing the CRF, we now could confirm that many neurons in primary visual cortex are indeed under the control of interhemispheric input through a response gain mechanism. This leads to a picture where the two hemispheres reinforce each other along the vertical midline of the visual field to compensate for the lack of input from the contralateral side (Peiker et al., 2013). However, we also found neurons whose contrast sensitivity was modulated by the interhemispheric network. Such an input gain modulation effectively multiplies the stimulus contrast that reaches a neuron. As a result, cortical neurons can adapt their dynamic range to the prevailing contrast levels in the visual stimulus (Ohzawa et al., 1982). It was shown previously that this contrast gain control is a dynamic process that involves the surrounding pool of neurons, probably mediated through longrange horizontal connections (Reynaud et al., 2012). The results presented here suggest that along the vertical meridian representation, the interhemispheric network takes over the role of the intrinsic long-range connections in setting the contrast gain of the local neuronal population. It is important to note that for many neurons we not only observed exclusive contrast or response gain modulations, but also a mixture of both. This indicates that the interhemispheric network influences a given neuron by more than one mechanism.

\section{Mechanisms of contrast and response gain}

For many recording sites, we found a mixture of contrast and response gain modulation. What could be the mechanism implementing both types of modulation in the brain? One possibility is that a given cortical neuron is affected by different callosal networks. In our model, we implemented this as a direct callosal input leading to a contrast gain and an indirect callosal input leading to a response gain. The relative contribution of each input will determine the outcome for a particular neuron.

To realize a contrast gain, the callosal input has to increase the firing rate at an intermediate contrast, but with no effect at low and high contrasts. A solution to this problem is the weighting of incoming activity by a contrast-dependent gain function (Cardin et al., 2008). This function is constant across orientations, preserving the multiplicative scaling of orientation tuning curves without changes in selectivity, as observed experimentally. Such a gain function could be the result of the biophysical properties of the receiving neurons (Cardin et al., 2008) or a population effect through divisive normalization (Albrecht and Hamilton, 1982; Heeger, 1992; Ohshiro et al., 2011; Carandini and Heeger, 2012). A third mechanism could be the targeting of specific compartments of callosal recipient cells, leading to nonlinear response modulations, as observed for subpopulations of inhibitory neurons (Wilson et al., 2012).

As discussed above, it seems unlikely that the strong rate decreases we observed on most recording sites are the sole contribution of the input through callosal synapses. In the model, we therefore implemented a second ICI, leading to a response gain. The ICI may come from a local population of neurons which themselves receive input from different callosal fibers, amplify it, and mix it together with the input from other sources, e.g., feedforward, feedback, or intrinsic horizontal connections. In this situation, the callosum just contributes to the pool of excitatory inputs, and therefore leads to an unspecific gain change. The latter mechanism might be generic to many cortical circuits and has been observed under different circumstances.
However, it is important to note that the input, which comes through the callosal connections, is by itself dependent on the properties of the visual stimulus. This is supported by in vivo studies showing that the visual callosal system links neurons with a similar response to stimulus contrast (Berardi et al., 1987; Tardif et al., 1997) and orientation (Schmidt et al., 1997; Rochefort et al., 2009). In this situation, the simple addition of the visually driven selective ipsilateral and contralateral inputs leads to a multiplicative gain change, as shown in our model. This is different from a scenario where the independent variable leading to a gain modulation of cortical responses is a scalar value. In the study by Olsen et al. (2012), for example, a fixed optogenetic activation of a pool of layer 6 pyramidal neurons led to a robust gain modulation of visually induced activity in the other cortical layers. New optogenetic tools may allow more specific stimulation or inhibition of subpopulations of callosal neurons, and thereby more insight into their interactions with local intrinsic activity.

\section{Factors determining the scaling mechanism}

If the relative contribution of direct callosal input and indirect network influences determines the scaling mechanism, this balance is probably reflected in the cellular and anatomical properties of a particular neuron. We tested for a range of receptive field, $\mathrm{CRF}$, and tuning properties, but did not find a strong correlation with any of those (Fig. 5). One exception was clearly the tendency toward a response gain for neurons with a steeper CRF. In a previous modeling study, it was proposed that the shape of the CRF is determined by the relative contributions of single neuron and network properties (Persi et al., 2011). Following this argumentation, our experimental results indicate that this relative contribution is also reflected in the connectivity profile of interhemispheric projections.

We also found the contrast gain mechanism to be particularly strong in the supragranular layers. This strengthens our hypothesis that a contrast gain modulation reflects a direct action of the callosal input, because most visual callosal connections terminate in these layers (Houzel et al., 1994).

Some neurons increased their activity after blocking callosal input. Those increases occurred mainly through a response gain mechanism (Fig. 3B). This indicates that those neurons were released from suppression by the indirect callosal input (i.e., response gain modulation) or that there was a more direct effect through interhemispheric feedforward inhibition (Toyama et al., 1974; Martin et al., 1983) targeting parvalbumin-positive interneurons known to mediate a response gain modulation (Wilson et al., 2012). Thus, our results support the hypothesis that modulations by a contrast or response gain reflect the connectivity pattern of a particular neuron and the network it is embedded in.

In summary, we showed that the combination of two signal streams in the visual cortex can lead to distinct effects, dependent on a particular stimulus feature. Whereas orientation tuning seems to be invariant to a modulating input, sensitivity and responsiveness to stimulus contrast can be increased (or decreased). This extends the role of the lateral interhemispheric network from a pure gain setting to the specific enhancement of a particular feature, namely, contrast sensitivity. Given the similarity to results obtained for the intrinsic horizontal network, we propose that our findings can be generalized to other corticocortical connections, helping us to understand how different signals are combined in the brain. 


\section{References}

Albrecht DG, Hamilton DB (1982) Striate cortex of monkey and cat: contrast response function. J Neurophysiol 48:217-237. Medline

Angelucci A, Bressloff PC (2006) Contribution of feedforward, lateral and feedback connections to the classical receptive field center and extraclassical receptive field surround of primate V1 neurons. Prog Brain Res 154:93-120. CrossRef Medline

Ayaz A, Chance FS (2009) Gain modulation of neuronal responses by subtractive and divisive mechanisms of inhibition. J Neurophysiol 101:958968. Medline

Berardi N, Bisti S, Maffei L (1987) The transfer of visual information across the corpus callosum: spatial and temporal properties in the cat. J Physiol 384:619-632. CrossRef Medline

Bhattacharyya A, Veit J, Kretz R, Bondar I, Rainer G (2013) Basal forebrain activation controls contrast sensitivity in primary visual cortex. BMC Neurosci 14:55. CrossRef Medline

Carandini M, Heeger DJ (2012) Normalization as a canonical neural computation. Nat Rev Neurosci 13:51-62. CrossRef Medline

Cardin JA, Palmer LA, Contreras D (2008) Cellular mechanisms underlying stimulus-dependent gain modulation in primary visual cortex neurons in vivo. Neuron 59:150-160. CrossRef Medline

Cavanaugh JR, Bair W, Movshon JA (2002) Nature and interaction of signals from the receptive field center and surround in macaque V1 neurons. J Neurophysiol 88:2530-2546. CrossRef Medline

Conti F, Manzoni T (1994) The neurotransmitters and postsynaptic actions of callosally projecting neurons. Behav Brain Res 64:37-53. CrossRef Medline

Douglas RJ, Koch C, Mahowald M, Martin KA, Suarez HH (1995) Recurrent excitation in neocortical circuits. Science 269:981-985. CrossRef Medline

Fiorani M, Azzi JC, Soares JG, Gattass R (2013) Automatic mapping of visual cortex receptive fields: a fast and precise algorithm. J Neurosci Methods 221:112-126. CrossRef

Gilbert CD, Wiesel TN (1979) Morphology and intracortical projections of functionally characterised neurones in the cat visual cortex. Nature 280: 120-125. CrossRef Medline

Heeger DJ (1992) Normalization of cell responses in cat striate cortex. Vis Neurosci 9:181-197. Medline

Houzel JC, Milleret C, Innocenti G (1994) Morphology of callosal axons interconnecting areas 17 and 18 of the cat. Eur J Neurosci 6:898-917. CrossRef Medline

Hubel DH, Wiesel TN (1967) Cortical and callosal connections concerned with the vertical meridian of visual fields in the cat. J Neurophysiol 30: 1561-1573. Medline

Innocenti GM (1994) Some new trends in the study of the corpus callosum. Behav Brain Res 64:1-8. CrossRef

Jeffs J, Ichida JM, Federer F, Angelucci A (2009) Anatomical evidence for classical and extra-classical receptive field completion across the discontinuous horizontal meridian representation of primate area V2. Cereb Cortex 19:963-981. CrossRef Medline

Katzner S, Busse L, Carandini M (2011) GABAA inhibition controls response gain in visual cortex. J Neurosci 31:5931-5941. CrossRef Medline

Manger PR, Kiper D, Masiello I, Murillo L, Tettoni L, Hunyadi Z, Innocenti GM (2002) The representation of the visual field in three extrastriate areas of the ferret (Mustela putorius) and the relationship of retinotopy and field boundaries to callosal connectivity. Cereb Cortex 12:423-437. CrossRef Medline

Martin KA, Somogyi P, Whitteridge D (1983) Physiological and morphological properties of identified basket cells in the cat's visual cortex. Exp Brain Res 50:193-200. Medline

Mitzdorf U, Singer W (1979) Excitatory synaptic ensemble properties in the visual cortex of the macaque monkey: a current source density analysis of electrically evoked potentials. J Comp Neurol 187:71-83. CrossRef

Movshon JA, Thompson ID, Tolhurst DJ (1978) Spatial and temporal contrast sensitivity of neurones in areas 17 and 18 of the cat's visual cortex. J Physiol 283:101-120. CrossRef Medline

Murphy BK, Miller KD (2003) Multiplicative gain changes are induced by excitation or inhibition alone. J Neurosci 23:10040-10051. Medline
Ohzawa I, Sclar G, Freeman RD (1982) Contrast gain control in the cat visual cortex. Nature 298:266-268. CrossRef Medline

Ohshiro T, Angelaki DE, DeAngelis GC (2011) A normalization model of multisensory integration. Nat Neurosci 14:775-782. CrossRef Medline

Olsen SR, Bortone DS, Adesnik H, Scanziani M (2012) Gain control by layer six in cortical circuits of vision. Nature 483:47-52. CrossRef Medline

Payne BR, Siwek DF, Lomber SG (1991) Complex transcallosal interactions in visual cortex. Vis Neurosci 6:283-289. CrossRef Medline

Peiker C, Wunderle T, Eriksson D, Schmidt A, Schmidt KE (2013) An updated midline rule: visual callosal connections anticipate shape and motion in ongoing activity across the hemispheres. J Neurosci 33:1803618046. CrossRef Medline

Persi E, Hansel D, Nowak L, Barone P, van Vreeswijk C (2011) Power-law input-output transfer functions explain the contrast-response and tuning properties of neurons in visual cortex. PLoS Comput Biol 7:e1001078. CrossRef Medline

Porter LL, White EL (1986) Synaptic connections of callosal projection neurons in the vibrissal region of mouse primary motor cortex: an electron microscopic/horseradish peroxidase study. J Comp Neurol 248:573-587. CrossRef

Quiroga RQ, Nadasdy Z, Ben-Shaul Y (2004) Unsupervised spike detection and sorting with wavelets and superparamagnetic clustering. Neural Comput 16:1661-1687. CrossRef Medline

Reynaud A, Masson GS, Chavane F (2012) Dynamics of local input normalization result from balanced short- and long-range intracortical interactions in area V1. J Neurosci 32:12558-12569. CrossRef Medline

Reynolds JH, Pasternak T, Desimone R (2000) Attention increases sensitivity of V4 neurons. Neuron 26:703-714. CrossRef Medline

Rochefort NL, Buzás P, Quenech'du N, Koza A, Eysel UT, Milleret C, Kisvárday ZF (2009) Functional selectivity of interhemispheric connections in cat visual cortex. Cereb Cortex 19:2451-2465. CrossRef Medline

Schmidt KE (2013) The visual callosal connection: a connection like any other? Neural Plast 2013:397176. Medline

Schmidt KE, Kim DS, Singer W, Bonhoeffer T, Löwel S (1997) Functional specificity of long-range intrinsic and interhemispheric connections in the visual cortex of strabismic cats. J Neurosci 17:5480-5492. Medline

Schmidt KE, Lomber SG, Innocenti GM (2010) Specificity of neuronal responses in primary visual cortex is modulated by interhemispheric corticocortical input. Cereb Cortex 20:2776-2786. CrossRef Medline

Sclar G, Freeman RD (1982) Orientation selectivity in the cat's striate cortex is invariant with stimulus contrast. Exp Brain Res 46:457-461. CrossRef Medline

Silver RA (2010) Neuronal arithmetic. Nat Rev Neurosci 11:474-489. CrossRef Medline

Skottun BC, De Valois RL, Grosof DH, Movshon JA, Albrecht DG, Bonds AB (1991) Classifying simple and complex cells on the basis of response modulation. Vision Res 31:1079-1086. Medline

Sun JS, Li B, Ma MH, Diao YC (1994) Transcallosal circuitry revealed by blocking and disinhibiting callosal input in the cat. Vis Neurosci 11:189197. CrossRef Medline

Swindale NV (1998) Orientation tuning curves: empirical description and estimation of parameters. Biol Cybern 78:45-56. CrossRef Medline

Tardif E, Richer L, Bergeron A, Lepore F, Guillemot JP (1997) Spatial resolution and contrast sensitivity of single neurons in area 19 of split-chiasm cats: a comparison with primary visual cortex. Eur J Neurosci 9:19291939. CrossRef Medline

Toyama K, Matsunami K, Ono T, Tokashiki S (1974) An intracellular study of neuronal organization in the visual cortex. Exp Brain Res 21:45-66. Medline

Tusa RJ, Rosenquist AC, Palmer LA (1979) Retinotopic organization of areas 18 and 19 in the cat. J Comp Neurol 185:657-678. CrossRef

Williford T, Maunsell JH (2006) Effects of spatial attention on contrast response functions in macaque area V4. J Neurophysiol 96:40-54. CrossRef Medline

Wilson NR, Runyan CA, Wang FL, Sur M (2012) Division and subtraction by distinct cortical inhibitory networks in vivo. Nature 488:343-348. CrossRef Medline

Wunderle T, Eriksson D, Schmidt KE (2013) Multiplicative mechanism of lateral interactions revealed by controlling interhemispheric input. Cereb Cortex 23:900-912. CrossRef Medline 